\title{
The Saddle Point Method for the Integral of the Absolute Value of the Brownian Motion
}

\author{
Leonid Tolmatz ${ }^{\dagger}$ \\ School of Mathematics and Statistics, University of Newcastle Upon Tyne, NE1 7RU, UK. \\ leonid.tolmatz@newcastle.ac.uk
}

The distribution function of the integral of the absolute value of the Brownian motion was expressed by L.Takács in the form of various series. In the present paper we determine the exact tail asymptotics of this distribution function. The proposed method is applicable to a variety of other Wiener functionals as well.

Keywords: Brownian motion, distribution, moments, asymptotics, saddle point, Airy functions.

\section{Introduction}

Let $B(t), t>0$, denote the standard Brownian motion. The interest to the functional

$$
\int_{0}^{1}|B(t)| d t
$$

is motivated by applications in order statistics: its distribution naturally appears in the consideration of the reliability of the procedure of determination of a theoretical distribution by drawing of a random sample. Namely, in the case when the sample size $N$ is a Poissonian random variable, see Kac (1949a). The distribution function of the functional (1) was determined explicitly by Takács (1993) in the form of various series. The logarithmic tail asymptotics of this distribution follows from independent results by Borell (1975) and Kallianpur and Oodaira (1978).

The purpose of the present paper is to outline how the saddle point method for integrals in the complex plane can be applied to determine the exact tail asymptotics of this distribution. These results where obtained in Tolmatz (1992), but remained unpublished. In the recent years the interest to distributions generated by various Wiener functionals is steadily growing; in particular we can mention applications to running times of certain algorithms and the applications in mathematical finance. Recently the asymptotics in question was computed by Fatalov (2003) by a completely different method.

The corresponding results for the Brownian bridge in the $L_{1}$ norm can be found in Cifarelli (1975), Shepp (1982), Johnson and Killeen (1983) and Tolmatz (2000). The method applied in Tolmatz (2000) is similar to the one used in Tolmatz (1992), but the case of Brownian motion is technically more complicated than that of the Brownian bridge, that is Tolmatz (2000) is a simplified version of Tolmatz (1992).

On results regarding the Brownian bridge and Brownian motion in the $L_{2}$ norm see Anderson and Darling (1952), Kiefer (1959), Tolmatz (2002), (2003), Cameron and Martin (1944).

\footnotetext{
${ }^{\dagger}$ This work was partly supported by the EPSRS, Grant no. GR/R71719/01. 


\section{Preliminaries}

Notation and some reference formulas. $A i(z), B i(z)$ denote the two Airy functions, $\zeta=2 / 3 z^{3 / 2}$; all fractional powers of complex numbers are taken as their principal values.

We shall make use the following asymptotic formulas for $A i(z)$ and $A i^{\prime}(z)$, see Olver (1974) Ch. 11:

$$
\begin{aligned}
A i(z) & =\frac{1}{2} \pi^{-1 / 2} z^{-1 / 4} e^{-\zeta}[1+O(1 / \zeta)], \text { in }|\arg z| \leq \pi-\delta \\
A i^{\prime}(z) & =-\frac{1}{2} \pi^{-1 / 2} z^{1 / 4} e^{-\zeta}[1+O(1 / \zeta)], \text { in }|\arg z| \leq \pi-\delta
\end{aligned}
$$

Let $\sigma(\lambda, t)$ denote the distribution we wish to determine,

$$
\sigma(\lambda, t)=\operatorname{Prob}\left\{\int_{0}^{t}|B(s)| d s<\lambda\right\},
$$

and $F(p, q)$ denotes its Laplace-Stieltjes transform with respect to $\lambda, t$ correspondingly. Similarly, let

$$
\sigma_{1}(\lambda, t, x)=\operatorname{Prob}\left\{\int_{0}^{t}|B(s)| d s<\lambda \mid B(t)=x\right\},
$$

and $F_{1}(p, q, x)$ be its Laplace-Stieltjes transform with respect to $\lambda, t$.

A direct application of the classical result of Kac (1949b), see also Rosenblatt (1951), yields:

$$
F_{1}(p, q, x)=\int_{0}^{+\infty} \int_{0}^{+\infty} e^{-p \lambda-q t} d_{\lambda} \sigma_{1}(\lambda, t, x) d t=-(2 p)^{-1 / 3} \frac{A i\left(2^{1 / 3} p^{-2 / 3}(q+p|x|)\right)}{A i^{\prime}\left(2^{1 / 3} p^{-2 / 3} q\right)}
$$

with $p$ and $q$ in the right half-plane. For $p, q>0$ it holds

$$
F(p, q)=2 \int_{0}^{+\infty} F_{1}(p, q, x) d x=-\frac{2 \cdot(2 p)^{-1 / 3}}{A i^{\prime}\left(2^{1 / 3} p^{-2 / 3} q\right)} \int_{0}^{\infty} A i\left(2^{1 / 3} p^{-2 / 3}(q+p x)\right) d x .
$$

\section{Some Analytical Properties of $F(p, q)$}

Lemma 1. Let $p, q>0$. Then $F(p, q)$ can be represented in the form

$$
F(p, q)=-\frac{2 \cdot(2 p)^{-1 / 3}}{A i^{\prime}\left(z_{0}(p, q)\right)}\left(\int_{0}^{z_{0}(p, q)} A i(z) d z-\alpha\right)
$$

where $z_{0}(p, q)=2^{1 / 3} p^{-2 / 3} q$, and

$$
\alpha=\int_{0}^{\infty} A i(z) d z=\frac{1}{3}
$$

Proof. In the integral in (7) we apply the change of variable $z=2^{1 / 3} p^{-2 / 3}(q+p x)$, which yields

$$
F(p, q)=-\frac{2 \cdot(2 p)^{-1 / 3}}{A i^{\prime}\left(z_{0}(p, q)\right)}\left(\int_{0}^{z_{0}(p, q)} A i(z) d z-\int_{0}^{+\infty} A i(z) d z\right)
$$


According to Prudnikov et. al. (1990), 2.11.2 p.125, we have:

$$
\int_{0}^{+\infty} \operatorname{Ai}(x) d x=\frac{3^{-1 / 2}}{2 \pi} \Gamma(1 / 3) \Gamma(2 / 3)
$$

which implies (9), and (8) follows.

Theorem 1. $F(p, q)$ in (7) admits an analytic continuation into the complex domain $\mathfrak{R} p>0, \mathfrak{R}>0$. In particular, such an analytic continuation is provided by the integral

$$
F(p, q)=-\frac{2 \cdot(2 p)^{-1 / 3}}{A i^{\prime}\left(z_{0}(p, q)\right)}\left(\int_{0}^{z_{0}(p, q)} A i(z) d z-\alpha\right)
$$

where the integration is taken along any smooth path in the right half-plane.

Proof. We observe that if $p$ and $q$ are in the right half-plane, then

$$
\left|\arg z_{0}(p, q)\right| \leq 2 / 3|\arg p|+|\arg q|<5 / 6 \pi
$$

All zeros of $A i^{\prime}(z)$ are real and negative; this fact together with (13) implies the theorem.

\section{Laplace Inversions}

Theorem 2. Let $F(p, q)$ be the analytical function as defined in Theorem 1. Then the distribution function $\sigma(\lambda, t)$ and its density function $f(\lambda, t)$ are given by the following double Laplace inversions:

$$
\begin{gathered}
\sigma(\lambda, t)=\frac{1}{(2 \pi i)^{2}} \int_{a-i \infty}^{a+i \infty} d q e^{q t} \int_{b-i \infty}^{b+i \infty} \frac{1}{p} F(p, q) e^{p \lambda} d p \\
f(\lambda, t)=\frac{1}{(2 \pi i)^{2}} \int_{a-i \infty}^{a+i \infty} d q e^{q t} \int_{b-i \infty}^{b+i \infty} F(p, q) e^{p \lambda} d p
\end{gathered}
$$

with any $a>0, b>0$.

Proof. The proof follows via standard manipulations with Laplace's integrals. The factor $\frac{1}{p}$ in (14) stems from a well known property of the Laplace transform: if $f(x)=\bar{f}(p)$, then $\int_{0}^{x} f(t) d t=\frac{1}{p} \bar{f}(p)$.

In the remaining part of the paper we shall treat $F(p, q)$ similarly to the treatment of $\tilde{F}(p, q)$ in Tolmatz (2000). However, the present situation with $F(p, q)$ requires more attention than it was in the case of $\tilde{F}(p, q)$ in the cited paper, because now $F(p, q)$ is no more given directly in terms of Airy functions, but it is given rather in terms of an integral of an Airy function, where the upper limit of integration itself depends on $p$ and $q$. 


\section{More on Analytical Properties of $F(p, q)$}

Let $a, b>0$ be fixed; we also fix an arbitrary complex $q$, such that $\Re q=a$. We denote $\theta=\arg q$; let $D_{\theta}$ be the complex $p$-plane with a cut along the ray

$$
p=r e^{i(\theta+\pi)}
$$

where $r \geq 0$ and $|\theta|<\pi / 2$. Any $p$ in $D_{\theta}$ has a unique representation in the form $p=\rho e^{i(\theta+\varphi)}$, where $\rho=|p|,|\varphi|<\pi$. For such a $p$ and $\beta$ real, the corresponding fractional power we define as $p^{\beta}=\rho^{\beta} e^{i \beta(\theta+\varphi)}$; we observe that $p^{\beta}$ is analytic in $D_{\theta}$.

Lemma 2. $F(\cdot, q)$ admits an analytic continuation into $D_{\theta}$.

Proof. We observe that

$$
\left|\arg z_{0}(p, q)\right| \leq 1 / 3|\theta|+2 / 3|\varphi|<5 / 6 \pi
$$

thus $A i^{\prime}\left(z_{0}(p, q)\right)$ has no zeros in $D_{\theta}$, so that (12) provides a desired analytical continuation.

Lemma 3. For $p$ large, $p \in D_{\theta}$, we have, for some $C, \gamma>0$ :

$$
|F(p, q)|<C \cdot|p|^{-\gamma} \text {. }
$$

Proof. For $|p| \rightarrow \infty$ in $D_{\theta}, z_{0}(p, q)=2^{1 / 3} p^{-2 / 3} q \rightarrow 0$, hence $\int_{0}^{z_{0}(p, q)} A i(z) d z$ remains bounded. Since $\arg z_{0}(p, q)<5 / 6 \pi, A i^{\prime}\left(z_{0}(p, q)\right)$ also remains bounded and bounded away from 0 ; thus

$$
|F(p, q)| \leq \frac{2|2 p|^{-2 / 3}}{\left|A i^{\prime}\left(z_{0}(p, q)\right)\right|}\left(\left|\int_{0}^{z_{0}(p, q)} A i(z) d z\right|+\alpha\right)<C \cdot|p|^{-2 / 3}
$$

and the Lemma follows with $\gamma=2 / 3$.

Lemma 4. The inner integral in (14),

$$
\int_{b-i \infty}^{b+i \infty} \frac{1}{p} F(p, q) e^{p \lambda} d p
$$

is well defined.

Proof. By Lemma 3,

$$
\left|\int_{b-i \infty}^{b+i \infty} \frac{1}{p} F(p, q) e^{p \lambda} d p\right|<\int_{b-i \infty}^{b+i \infty} \frac{C}{|p|^{1+\gamma}} e^{b \lambda}|d p|<\infty .
$$




\section{Transformation of the Contours}

In this section we show that the vertical path of integration in the integral $\int_{b-i \infty}^{b+i \infty} 1 /(p-i \varepsilon) F(p, q) e^{p \lambda} d p$ can be replaced by integration on the both sides of the cut along the ray (16), see Theorem 6 . A similar transformation of contours was applied in Tolmatz (2000).

It is worthwhile to mention that the choice of the contour of integration in the complex plane is the crucial in computations with saddle points. Even in the case of a one-dimensional complex integral, when we deal with a curve in the complex plane, finding a suitable contour is usually a non-trivial task, while here we are dealing with a triple complex integral.

For any fixed $\theta \in\left(-\frac{\pi}{2}, \frac{\pi}{2}\right)$, and $\delta \geq 0,0<r<\varepsilon<b<R$, we define

a closed contour $C_{\delta}$ in the complex $p$-plane as follows:

$$
\begin{aligned}
C_{\delta} & =C_{b} \cup C_{R}^{\delta} \cup C_{\delta}^{+} \cup C_{r}^{\delta} \cup C_{\delta}^{-} \\
\text {where } C_{b} & =\{p \mid \Re p=b \text { and }|p| \leq R\} \\
C_{R}^{\delta} & =\{p|\Re p \leq b,| p \mid=R, \text { and }|\arg p-\theta|<\pi-\delta\} \\
C_{r}^{\delta} & =\{p|\Re p \leq b,| p \mid=r, \text { and }|\arg p-\theta|<\pi-\delta\} \\
C_{\delta}^{ \pm} & =\{p \mid \arg p=\theta \pm \pi \mp \delta \text { and } r \leq|p| \leq R\}
\end{aligned}
$$

Let $D_{\delta}$ denote the domain bounded by $C_{\delta}$, and $\bar{D}_{\delta}$ its closure. We shall denote $f_{\varepsilon}(p)=\frac{1}{p-i \varepsilon} F(p, q) e^{p \lambda}$; by Lemma $2, f_{\varepsilon}(p)$ is analytic in $D_{\theta}$, except the pole at $p=i \varepsilon$. It is easy to see that

$$
\int_{C_{\delta}} f_{\varepsilon}(p) d p=2 \pi i \operatorname{Res}_{p=i \varepsilon} f_{\varepsilon}(p) .
$$

We shall need the following preleminary results:

\section{Lemma 5.}

$$
\lim _{\varepsilon \rightarrow 0} \int_{b-i \infty}^{b+i \infty} f_{\varepsilon}(p) d p=\lim _{\varepsilon \rightarrow 0} \int_{b-i \infty}^{b+i \infty} \frac{F(p, q)}{p-i \varepsilon} e^{p \lambda} d p=\int_{b-i \infty}^{b+i \infty} \frac{F(p, q)}{p} e^{p \lambda} d p
$$

Proof. We have with making use of Lemma 3:

$$
\left|\int_{b-i \infty}^{b+i \infty}\left(\frac{1}{p-i \varepsilon}-\frac{1}{p}\right) F(p, q) e^{p \lambda} d p\right|=\varepsilon\left|\int_{b-i \infty}^{b+i \infty} \frac{1}{p(p-i \varepsilon)} F(p, q) e^{p \lambda} d p\right| \rightarrow 0 .
$$

Let us denote

$$
\begin{aligned}
& p=\rho e^{i(\varphi+\theta)} \\
& z^{ \pm}(\rho)=2^{1 / 3} \rho^{-2 / 3} e^{-2 / 3(\theta \pm \pi) i} q \\
& f_{\varepsilon}^{ \pm}(\rho)=\frac{-\exp \left(-\rho e^{i \theta} \lambda\right)}{\rho e^{i \theta}+i \varepsilon} \frac{z^{ \pm}(\rho) e^{i \theta}}{A i^{\prime}\left(z^{ \pm}(\rho)\right)} \frac{1}{q}\left(\int_{0}^{z^{ \pm}(\rho)} A i(z) d z-\alpha\right)
\end{aligned}
$$


Lemma 6 (The passage $\delta \rightarrow 0$ in (29)).

$$
\begin{aligned}
& \int_{r}^{R} f_{\varepsilon}^{+}(\rho) d \rho-\int_{r}^{R} f_{\varepsilon}^{-}(\rho) d \rho+ \\
& \int_{C_{b}} f_{\varepsilon}(p) d p+\int_{C_{R}^{0}} f_{\varepsilon}(p) d p+\int_{C_{r}^{0}} f_{\varepsilon}(p) d p= \\
& 2 \pi i \operatorname{Res}_{p=i \varepsilon} f_{\varepsilon}(p) .
\end{aligned}
$$

Proof. The proof follows by an elementary justifiable termwise passage to the limit $\delta \rightarrow 0$ in the equation

$$
\int_{C_{\delta}} f_{\varepsilon}(p) d p=\left(\int_{C_{b}}+\int_{C_{R}^{\delta}}+\int_{C_{r}^{\delta}}+\int_{C_{\delta}^{+}}+\int_{C_{\delta}^{-}}\right) f_{\varepsilon}(p) d p
$$

\section{Theorem 3.}

$$
\int_{b-i \infty}^{b+i \infty} f_{\varepsilon}(p) d p=2 \pi i \operatorname{Res}_{p=i \varepsilon} f_{\varepsilon}(p)-\int_{0}^{+\infty}\left(f_{\varepsilon}^{+}(\rho)-f_{\varepsilon}^{-}(\rho)\right) d \rho .
$$

Proof. Let $R \rightarrow+\infty$ in (33). By Lemma 3 we can see that $\int_{C_{R}^{0}} f_{\varepsilon}(p) d p \rightarrow 0$; this yields

$$
\int_{r}^{+\infty}\left(f_{\varepsilon}^{+}(\rho)-f_{\varepsilon}^{-}(\rho)\right) d \rho+\int_{b-i \infty}^{b+i \infty} f_{\varepsilon}(p) d p+\int_{C_{r}^{0}} f_{\varepsilon}(p) d p=2 \pi i \operatorname{Res}_{p=i \varepsilon} f_{\varepsilon}(p) .
$$

To complete the proof, it remains to show that in (36)

$$
\int_{C_{r}^{0}} \rightarrow 0 \quad \text { as } r \rightarrow 0 .
$$

We can write

$$
-\int_{0}^{z_{0}(p, q)} A i(z) d z+\alpha=\int_{z_{0}(p, q)}^{0} A i(z) d z+\int_{0}^{+\infty} A i(z) d z=\int_{C_{1}(p, q)} A i(z) d z
$$

where the path $C_{1}(p, q)$ is the broken line, which is defined by its three vertices: $z=z_{0}(p, q), z=0$, and $z=+\infty$. (That is $C_{1}(p, q)$ proceeds from $z_{0}(p, q)$ to the origin, and then along the real axis).

It is not difficult to see, that $C_{1}(p, q)$ is equivalent to $C_{2}(p, q)$, where $C_{2}(p, q)$ is the ray

$$
C_{2}(p, q)=\left\{z \mid z=z_{0}(p, q)+\tau, \tau \geq 0\right\}
$$

that is we have

$$
\int_{C_{1}(p, q)} A i(z) d z=\int_{C_{2}(p, q)} A i(z) d z
$$

(To show this, we apply Cauchy's theorem with the asymptotc estimate $A i(z)=1 /(2 \sqrt{\pi}) z^{-1 / 4} \exp (-\zeta)(1+$ $O(1 / \zeta))$, where $\left.\zeta=2 / 3 z^{3 / 2}\right)$. Denote $S=\{z|| \arg z \mid<5 / 6 \pi\}$. Since $\left|z_{0}(p, q)\right| \rightarrow \infty$ as $p \rightarrow 0$, we wish to estimate

$$
-\frac{1}{A i^{\prime}\left(z_{0}(p, q)\right)}\left(\int_{0}^{z_{0}(p, q)} A i(z) d z-\alpha\right)=\frac{1}{A i^{\prime}\left(z_{0}(p, q)\right)} \int_{C_{2}(p, q)} A i(z) d z
$$


when $z_{0}(p, q)$ is large, under the condition $p \in D_{\theta}$; in such a case $z_{0}(p, q) \in S$; moreover, $C_{2}(p, q) \subset S$.

Denote

$$
I\left(z_{0}(p, q)\right)=\int_{C_{2}(p, q)} A i(z) d z=\int_{0}^{+\infty} A i\left(z_{0}(p, q)+\tau\right) d \tau
$$

From

$$
|A i(z)|<\frac{C\left|e^{-\zeta}\right|}{1+|z|^{1 / 4}}<C\left|e^{-\zeta}\right|
$$

and

$$
\left|1 / A i^{\prime}\left(z_{0}(p, q)\right)\right|<\frac{C\left|e^{\zeta_{0}(p, q)}\right|}{1+\left|z_{0}(p, q)\right|^{1 / 4}}<C\left|e^{\zeta_{0}(p, q)}\right|,
$$

where $\zeta_{0}(p, q) \equiv 2 / 3 z_{0}^{3 / 2}(p, q)$ and $z_{0}(p, q) \in S$, we obtain

$$
\begin{aligned}
& \left|I\left(z_{0}(p, q)\right) / \operatorname{Ai}\left(z_{0}(p, q)\right)\right|<\int_{0}^{+\infty}\left|\operatorname{Ai}\left(z_{0}(p, q)+\tau\right)\right| /\left|A i\left(z_{0}(p, q)\right)\right| d \tau< \\
& C \int_{0}^{+\infty}\left|\exp \left\{-2 / 3\left(\left[z_{0}(p, q)+\tau\right)^{3 / 2}-z_{0}(p, q)^{3 / 2}\right]\right\}\right| d \tau,
\end{aligned}
$$

that is

$$
\left|I\left(z_{0}(p, q)\right) / A i\left(z_{0}(p, q)\right)\right|<C \int_{0}^{+\infty} \exp \left(-\int_{0}^{\tau} \Re\left(\sqrt{z_{0}(p, q)+s}\right) d s\right) d \tau .
$$

It holds $\left|\arg z_{0}(p, q)+s\right|<5 / 6 \pi$, so we have $\cos \left[1 / 2\left(\arg z_{0}(p, q)+s\right)\right]>\cos (5 / 12 \pi) \equiv \gamma_{1}>0$, and $\Re \sqrt{z_{0}(p, q)+s}>\left|z_{0}(p, q)+s\right|^{1 / 2} \gamma_{1}$. Denote $\Delta=\min _{s \geq 0}\left|z_{0}(p, q)+s\right|$. We observe that

$$
\Delta=\left\{\begin{array}{l}
\left|z_{0}(p, q)\right|, \text { if } \Re z_{0}(p, q) \geq 0, \\
\mathfrak{I} z_{0}(p, q), \text { if } \Re z_{0}(p, q)<0 .
\end{array}\right.
$$

An elementary consideration shows that if $\left|z_{0}(p, q)\right|>1$, then

$$
\Re \sqrt{z_{0}(p, q)+s}>\left|z_{0}(p, q)+s\right|^{1 / 2} \gamma_{1}>\Delta^{1 / 2} \gamma_{1}>\left|z_{0}(p, q)\right|^{1 / 4} \gamma,
$$

with some $\gamma>0$. From this, if $\left|z_{0}(p, q)\right|>1$, then

$$
\int_{0}^{\tau} \Re \sqrt{z_{0}(p, q)+s} d s>\left|z_{0}(p, q)\right|^{1 / 4} \gamma \tau .
$$

We substitute (50) in (47) and obtain

$$
\left|I\left(z_{0}(p, q)\right) / A i\left(z_{0}(p, q)\right)\right|<C \int_{0}^{+\infty} \exp \left(-\left|z_{0}(p, q)\right|^{1 / 4} \gamma \tau\right) d \tau=\frac{C}{\left|z_{0}(p, q)\right|^{1 / 4} \gamma},
$$

that is the left-hand side of (51) is bounded for for $\left|z_{0}(p, q)\right|>1$ and $z_{0}(p, q) \in S$. From this, with $|p|=r$, we obtain

$$
\left|f_{\varepsilon}(p)\right|=\left|\frac{1}{p-i \varepsilon}\right||F(p, q)|\left|e^{p \lambda}\right|=\left|\frac{1}{p-i \varepsilon}\right|\left|\frac{2}{(2 p)^{2 / 3}}\right|\left|I\left(z_{0}(p, q)\right) / A i\left(z_{0}(p, q)\right) \| e^{p \lambda}\right|<C r^{-2 / 3},
$$


where $C$ is a constant; therefore

$$
\left|\int_{C_{r}^{0}} f_{\varepsilon}(p) d p\right|<2 \pi C r^{1 / 3} \rightarrow 0 \text { as } r \rightarrow 0,
$$

and (37) follows.

\section{Further Transformations}

Remark. In the remaining part of this paper some technical lemmas will be given without proofs. The reader can either treat these as exercises, or find proofs in Tolmatz (1992).

We shall denote

$$
J_{\varepsilon}(\lambda, \theta)=\int_{0}^{+\infty}\left(f_{\varepsilon}^{+}(\rho)-f_{\varepsilon}^{-}(\rho)\right) d \rho
$$

this integral has appeared in the statement of Theorem 3. Let also

$$
\xi_{0}(\rho) \equiv 2^{1 / 3} \rho^{-2 / 3} \exp (-2 \theta i / 3) q .
$$

Then

$$
z^{ \pm}(\rho)=\exp (\mp 2 \pi i / 3) \xi_{0}(\rho)
$$

Notice that

$$
\left|\xi_{0}(\rho)\right|=\left|z^{ \pm}(\rho)\right| \quad \text { and } \quad \arg \xi_{0}(\rho)=1 / 3 \theta
$$

In the estimates to follow $C, C_{1} \ldots$ and $\gamma, \gamma_{1} \ldots$ denote some positive constants.

\section{Theorem 4.}

$$
J_{\varepsilon}(\lambda, \theta)=\int_{0}^{+\infty} d \rho \cdot k(\rho)\left(\alpha B_{1}(\rho)+\int_{0}^{\xi_{0}(\rho)} B_{2}(\xi) d \xi\right),
$$

where the notation is:

$$
\begin{aligned}
& k(\rho)=-\frac{2^{1 / 3} \rho^{-2 / 3} \exp (i \theta / 3)}{\rho \exp (i \theta)+i \varepsilon} \exp \left(-\rho e^{i \theta} \lambda\right), \\
& B_{1}(\rho)=\frac{e^{2 \pi i / 3}}{A i^{\prime}\left(e^{2 \pi i / 3} \xi_{0}(\rho)\right)}-\frac{e^{-2 \pi i / 3}}{A i^{\prime}\left(e^{-2 \pi i / 3} \xi_{0}(\rho)\right)}, \\
& B_{2}(\rho, \xi)=\frac{e^{\pi i / 3} A i\left(e^{2 \pi i / 3} \xi\right)}{A i^{\prime}\left(e^{2 \pi i / 3} \xi_{0}(\rho)\right)}-\frac{e^{-\pi i / 3} A i\left(e^{-2 \pi i / 3} \xi\right)}{A i^{\prime}\left(e^{-2 \pi i / 3} \xi_{0}(\rho)\right)} .
\end{aligned}
$$

Proof. By (32) we have

$$
\begin{aligned}
f_{\varepsilon}^{+}(\rho)-f_{\varepsilon}^{-}(\rho) & =\frac{\exp \left(-\rho e^{i \theta} \lambda\right)}{\rho e^{i \theta}+i \varepsilon} \frac{e^{i \theta}}{q}\left[\frac{z^{+}(\rho)}{A i^{\prime}\left(z^{+}(\rho)\right)} \int_{z^{+}(\rho)}^{0} A i(z) d z-\right. \\
& -\frac{z^{-}(\rho)}{A i^{\prime}\left(z^{-}(\rho)\right)} \int_{z^{-}(\rho)}^{0} A i(z) d z+ \\
& \left.+\alpha\left(\frac{z^{+}(\rho)}{A i^{\prime}\left(z^{+}(\rho)\right)}-\frac{z^{-}(\rho)}{A i^{\prime}\left(z^{-}(\rho)\right)}\right)\right] .
\end{aligned}
$$


The reparametrization

$$
\int_{z^{ \pm}(\rho)}^{0} A i(z) d z=e^{\mp 2 \pi i / 3} \int_{\xi_{0}(\rho)}^{0} A i\left(e^{\mp 2 \pi i / 3} \xi\right) d \xi
$$

and obvious substitutions yield (58).

We shall need the identity (Olver (1974) Ch.11)

$$
A i\left(z e^{ \pm 2 \pi i / 3}\right)=\frac{1}{2} e^{ \pm \pi i / 3}[A i(z) \mp i B i(z)]
$$

differentiation of which yields

$$
A i^{\prime}\left(z e^{ \pm 2 \pi i / 3}\right)=\frac{1}{2} e^{\mp \pi i / 3}\left[A i^{\prime}(z) \mp i B i^{\prime}(z)\right]
$$

Lemma 7 (Transformation of $B_{1}(\rho)$ ).

$$
B_{1}(\rho)=-4 i / B i^{\prime}\left(\xi_{0}(\rho)\right)\left(1+\beta_{1}(\rho)\right),
$$

where $\beta_{1}(\rho)$ is some function such that

$$
\left|\beta_{1}(\rho)\right|<C_{1} \exp \left(-\gamma_{1}\left|\xi_{0}(\rho)\right|^{3 / 2}\right) .
$$

Lemma 8 (Transformation of $\left.\int B_{2}(\rho, \xi) d \xi\right)$.

$$
\alpha B_{1}(\rho)+\int_{0}^{\xi_{0}(\rho)} B_{2}(\rho, \xi) d \xi=-6 i \alpha / B i^{\prime}\left(\xi_{0}(\rho)\right)(1+\beta(\rho))
$$

where $\beta(\rho)$ is some function such that

$$
|\beta(\rho)|<C \exp \left(-\gamma\left|\xi_{0}(\rho)\right|^{3 / 2}\right)
$$

Theorem 5.

$$
J_{\varepsilon}(\lambda, \theta)=\int_{0}^{+\infty} d \rho \cdot \frac{6 \cdot 2^{1 / 3} \rho^{-2 / 3} e^{i \theta / 3}}{\rho e^{i \theta}+i \varepsilon} \exp \left(-\rho e^{i \theta} \lambda\right) \frac{i \alpha(1+\beta(\rho))}{B i^{\prime}\left(\xi_{0}(\rho)\right)},
$$

where, for some $C, \gamma>0$ :

$$
|\beta(\rho)|<C \exp \left(-\gamma\left|\xi_{0}(\rho)\right|^{3 / 2}\right)
$$

Proof. The proof follows from (58) and the previous lemma. 
8 The Passage $\varepsilon \rightarrow 0$.

\section{Lemma 9.}

$$
\begin{aligned}
& \lim _{\varepsilon \rightarrow 0} J_{\varepsilon}(\lambda, \theta)= \\
& 6 \cdot 2^{1 / 3} \alpha i \int_{0}^{+\infty} d \rho \cdot \rho^{-5 / 3} \exp (-4 / 3 \theta i) \exp \left(-\rho e^{i \theta} \lambda\right) \cdot 1 / B i^{\prime}\left(\xi_{0}(\rho)\right) \cdot(1+\beta) .
\end{aligned}
$$

We shall use the notation

$$
J(\lambda, \theta)=\lim _{\varepsilon \rightarrow 0} J_{\varepsilon}(\lambda, \theta)
$$

Lemma 10.

$$
\lim _{\varepsilon \rightarrow 0} \operatorname{Res}_{p=i \varepsilon} f_{\varepsilon}(p)=\frac{1}{q} .
$$

The following theorem sums up the results of previous sections.

Theorem 6.

$$
\begin{aligned}
& \int_{b-\infty}^{b+\infty} \frac{1}{p} F(p, q) e^{p \lambda} d p= \\
& \frac{2 \pi i}{q}-6 \cdot 2^{1 / 3} \alpha i \int_{0}^{+\infty} \frac{\rho^{-5 / 3} \exp (-4 / 3 \theta i) \exp \left(-\rho e^{i \theta} \lambda\right)}{B i^{\prime}\left(\xi_{0}(\rho)\right)}(1+\beta(\rho)) d \rho
\end{aligned}
$$

where

$$
\begin{aligned}
\alpha & =\int_{0}^{+\infty} \operatorname{Ai}(x) d x=\frac{1}{3}, \\
\xi_{0}(\rho) & =2^{1 / 3} \rho^{-2 / 3} e^{-2 \theta i / 3} q, \\
\beta(\rho) & =O\left(\exp \left(-\gamma\left|\xi_{0}(\rho)\right|^{3 / 2}\right)\right) .
\end{aligned}
$$

In the following we shall use formula (75) in the form

$$
\int_{b-\infty}^{b+\infty} \frac{1}{p} F(p, q) e^{p \lambda} d p=\frac{2 \pi i}{q}-6 \cdot 2^{1 / 3} \alpha i \int_{0}^{+\infty e^{i \theta}} \frac{p^{-5 / 3} \exp (-p \lambda)}{B i^{\prime}\left(2^{1 / 3} p^{-2 / 3} q\right)}(1+\beta(p)) d p
$$

where $\int_{0}^{+\infty e^{i \theta}}$ denotes an integral in the $p$-plane along the ray $r e^{i \theta}, r \geq 0$.

\section{Some Expressions for $\sigma(\lambda, t)$.}

For brevity we denote

$$
\mathbf{J}(\lambda, t)=\int_{a-i \infty}^{a-i \infty} d q e^{q t} \int_{0}^{+\infty e^{i \theta}} \frac{p^{-5 / 3} \exp (-p \lambda)}{B i^{\prime}\left(2^{1 / 3} p^{-2 / 3} q\right)}(1+\beta(p)) d p
$$

that is the distribution $\sigma(\lambda, t)$ and its tail $T(\lambda, t)$, according to (14) and (79) take the form

$$
\begin{aligned}
& \sigma(\lambda, t)=1+3 \cdot 2^{-2 / 3} \alpha i / \pi^{2} \mathbf{J}(\lambda, t), \\
& T(\lambda, t)=-3 \cdot 2^{-2 / 3} \alpha i / \pi^{2} \mathbf{J}(\lambda, t) .
\end{aligned}
$$


The substitution

$$
\left\{\begin{array}{l}
q=a(1+i \tan \theta) \\
p=r e^{i \theta}
\end{array}\right.
$$

where $|\theta|<\pi / 2, r>0$ transforms the integral $\mathbf{J}(\lambda, t)$ into the form

$$
\mathbf{J}(\lambda, t)=a i \int_{-\pi / 2}^{\pi / 2} \sec ^{2} \theta d \theta \int_{0}^{+\infty} g(r, \theta) r \exp (i \theta) d r
$$

where $g(r, \theta)$ is the result of substitution (83) into the integrand in (80).

Lemma 11. The double integral which corresponds to the repeated integral (84), converges absolutely, that is

$$
\int_{-\pi / 2}^{\pi / 2} \int_{0}^{+\infty}\left|\sec ^{2} \theta g(r, \theta) r \exp (i \theta)\right| d r d \theta<\infty
$$

\section{Application of Laplace's Method for Integrals}

We wish to apply to the integral $\mathbf{J}(\lambda, t)$ the saddle point method for integrals in a fashion similar to that in Tolmatz (2000).

\section{Proposition 12.}

$$
\mathbf{J}(\lambda, t)=2^{-1 / 12} \sqrt{\pi} \int_{a-i \infty}^{a+i \infty} d q \int_{0}^{+\infty \exp i \theta}(1+O(1 / \zeta)) p^{-3 / 2} q^{-1 / 4} \exp (-\Phi(p, q, \lambda, t)) d p
$$

where

$$
\Phi(p, q, \lambda, t)=2 \sqrt{2} / 3 q^{3 / 2} p^{-1}-q t+p \lambda
$$

Proof. We substitute in (80)

$$
\left.B i^{\prime}(z(p, q))\right)=z^{1 / 4}(p, q) \pi^{-1 / 2} \exp \zeta(p, q)\left(1+O\left(\frac{1}{\zeta(p, q)}\right)\right)
$$

and take into account that

$$
(1+\beta)\left(1+O\left(\frac{1}{\zeta(p, q)}\right)\right)=1+O\left(\frac{1}{\zeta(p, q)}\right) .
$$

One may expect that the system of equations

$$
\left\{\begin{array}{l}
\Phi_{p}=0 \\
\Phi_{q}=0
\end{array}\right.
$$

provides a suitable saddle point for the integrand in $\mathbf{J}(\lambda, t)$. We shall see that indeed this is the case. 
Proposition 13. The system of equations

$$
\left\{\begin{array}{l}
\Phi_{p}=-2 \sqrt{2} / 3 q^{3 / 2} p^{-2}+\lambda=0 \\
\Phi_{q}=\sqrt{2} q^{1 / 2} p^{-1}-t=0
\end{array}\right.
$$

has a unique (real) solution,

$$
\begin{gathered}
p=3 \lambda / t^{3}, \\
q=9 \lambda^{2} /\left(2 t^{4}\right) .
\end{gathered}
$$

In the following we shall assume in all the formulas $t=1$, that is all the expressions will relate to the functional

$$
\int_{0}^{1}\left|B_{s}\right| d s
$$

and the self-explanatory notation like $\mathbf{J}(\lambda)=\mathbf{J}(\lambda, 1)$ will be used.

Lemma 14. Let by definition $q_{0}=9 / 2$. Then

$$
\mathbf{J}(\lambda)=2^{-1 / 12} \sqrt{\pi} \lambda i \int_{-\pi / 2}^{\pi / 2} \int_{0}^{+\infty}\left(1+\beta^{\prime}\right) f(r, \theta) \exp \left(-\Phi_{0} \lambda^{2}\right) d r d \theta,
$$

where the notation is

$$
\begin{gathered}
\beta^{\prime}=O\left(r \cos ^{3 / 2} \theta \cdot \lambda^{-2}\right), \\
f(r, \theta)=q_{0}^{3 / 4} r^{-3 / 2} \exp (-i \theta / 2) \sec ^{2} \theta, \\
\Phi_{0}=\Phi_{0}(r, \theta)=9 \frac{e^{i \theta / 2}}{\cos ^{3 / 2} \theta} \frac{1}{r}-q_{0}(1+i \tan \theta)+r e^{i \theta} .
\end{gathered}
$$

We note that by Lemma 11 integral (95) converges absolutely. Lemma 14 implies that

$$
T(\lambda)=3 \cdot 2^{-2 / 3} \frac{\alpha}{\pi^{2}} 2^{-1 / 12} \sqrt{\pi} \lambda i \int_{-\pi / 2}^{\pi / 2} \int_{0}^{+\infty}\left(1+\beta^{\prime}\right) f(r, \theta) \exp \left(-\Phi_{0} \lambda^{2}\right) d r d \theta .
$$

Lemma 15. The function

$$
\Re \Phi_{0}(r, \theta)=9 \frac{\cos \theta / 2}{\cos ^{1 / 2} \theta} \cdot \frac{1}{r}+r \cos \theta-q_{0}
$$

attains its unique absolute minimum at $\left(r_{0}, \theta_{0}\right)=(3,0)$; the corresponding value is

$$
m=\mathfrak{R} \Phi_{0}(3,0)=3 / 2 .
$$

Let $S_{\delta}$ denote an open $\delta$-neighbourhood of $\left(r_{0}, 0\right)$ in $\mathbb{R}^{2}$, and let by definition

$$
D=\{(r, \theta)|0<r,| \theta \mid<\pi / 2\}
$$

then for the integral in (99) we have

$$
\int_{-\pi / 2}^{\pi / 2} \int_{0}^{+\infty}=\iint_{D}=\left(\iint_{S_{\delta}}+\iint_{D / S_{\delta}}\right)
$$


Lemma 16. With some $\varepsilon>0$, one has:

$$
\iint_{D / S_{\delta}}\left(1+\beta^{\prime}\right) f(r, \theta) \exp \left(-\Phi_{0} \lambda^{2}\right) d r d \theta=O\left(e^{-(m+\varepsilon) \lambda^{2}}\right),
$$

Lemma 16 shows that in order to determine an asymptotics of the tail $T(\lambda)$ it suffices to determine an asymptotics of the integral

$$
\mathbf{J}_{\delta}(\lambda) \equiv \iint_{S_{\delta}}\left(1+\beta^{\prime}\right) f(r, \theta) \exp \left(-\Phi_{0} \lambda^{2}\right) d r d \theta
$$

Instead of $r$ we shall use the local variable $\eta$, where $r=r_{0}+\eta$.

Lemma 17.

$$
\Phi_{0}(\eta, \theta)=\frac{3}{2}+\frac{1}{3} \eta^{2}+\frac{1}{2} i \eta \theta+\frac{3}{8} \theta^{2}+O\left(\left(\eta^{2}+\theta^{2}\right)^{1 / 2}\right) .
$$

Proof. Linear terms in (106) vanish by virtue of Lemma 15; the second order terms and the $O$-term result from a straightforward Taylor expansion.

Lemma 18.

$$
\mathbf{J}_{\delta}(\lambda)=2^{-3 / 4} 4 \pi / \sqrt{3} \lambda^{-2} \exp \left(-3 / 2 \lambda^{2}\right)(1+o(1))
$$

as $\lambda \rightarrow+\infty$.

Lemmas 16 and 18 imply the main result of the present paper.

Theorem 7. As $\lambda \rightarrow+\infty$, one has:

$$
\begin{gathered}
\sigma(\lambda)=1-\sqrt{\frac{2}{3 \pi}} \frac{1}{\lambda} e^{-3 \lambda^{2} / 2}(1+o(1)), \\
T(\lambda)=\sqrt{\frac{2}{3 \pi}} \frac{1}{\lambda} e^{-3 \lambda^{2} / 2}(1+o(1))
\end{gathered}
$$

A similar derivation yields the asymptotics

$$
f(\lambda)=\sqrt{\frac{6}{\pi}} e^{-3 \lambda^{2} / 2}(1+o(1))
$$

By the scaling property, for $t>0$ we obtain

$$
\sigma(\lambda, t)=\sigma\left(\lambda t^{-3 / 2}, 1\right)
$$

that is for any fixed $t>0$ and $\lambda \rightarrow+\infty$ we have the asymptotics

$$
\sigma(\lambda, t)=1-\sqrt{\frac{2}{3 \pi}} \frac{t^{3 / 2}}{\lambda} e^{-3 \lambda^{2} /\left(2 t^{3}\right)}(1+o(1)),
$$

and correspondingly

$$
f(\lambda, t)=\sqrt{\frac{6}{\pi}} t^{-3 / 2} e^{-3 \lambda^{2} /\left(2 t^{3}\right)}(1+o(1)) .
$$

Remark. The proposed method readily provides, for any fixed $t>0$, asymptotic expansions of $\sigma(\lambda, t)$ and $f(\lambda, t)$ in negative powers of $\lambda$ up to any order. 


\section{An Application to Moments}

The distribution function $F(\lambda)$ of the Brownian motion in the $L_{1}$ norm was determined by Takács (1993), and in the same paper the moments of this distribution $\mu_{r}$ were given as following:

$$
\mu_{r}=\frac{L_{r} r !}{2^{r / 2} \Gamma((3 r+2) / 2)}
$$

for $r=0,1, \ldots$, where $L_{0}=1$ and

$$
L_{n}=\beta_{n}+\sum_{j=1}^{n} \frac{6 j+1}{6 j-1} \alpha_{j} L_{n-j}
$$

for $n=1,2, \ldots$, where $\alpha_{0}=1$ and

$$
\alpha_{j}=\frac{\Gamma(3 j+1 / 2)}{(36)^{j} j ! \Gamma(j+1 / 2)}
$$

for $j=1,2, \ldots$, where $\beta_{0}=1$ and

$$
\beta_{k}=\alpha_{k}+\frac{3(2 k-1)}{4} \beta_{k-1}
$$

for $k=1,2, \ldots$. From the relationships (114)-(117) Takács derived the asymptotic formula

$$
\mu_{r} \sim \sqrt{2}\left(\frac{r}{3 e}\right)^{r / 2}
$$

as $r \rightarrow \infty$. The asymptotics (110) leads to an asymptotic formula for the moments $\mu_{r}$ in a different form which, in turn, implies (118).

\section{Theorem 8.}

$$
\mu_{r} \sim \frac{1}{\sqrt{\pi}}\left(\frac{2}{3}\right)^{r / 2} \Gamma((r+1) / 2) .
$$

Proof.

$$
\mu_{r}=\int_{0}^{+\infty} \lambda^{r} f(\lambda) d \lambda=\sqrt{\frac{6}{\pi}} \int_{0}^{+\infty} \lambda^{r} e^{-(3 / 2) \lambda^{2}}(1+o(1)) d \lambda=\frac{1}{\sqrt{\pi}}\left(\frac{2}{3}\right)^{r / 2} \Gamma\left(\frac{r+1}{2}\right)(1+o(1)) .
$$

From (119) and by Stirling's formula $\Gamma\left(\frac{r+1}{2}\right) \sim \sqrt{2 \pi} e^{-(r+1) / 2}\left(\frac{r+1}{2}\right)^{r / 2}$ we obtain $\mu_{r} \sim \sqrt{\frac{2}{e}}\left(\frac{r+1}{3 e}\right)^{r / 2}$ as $r \rightarrow \infty$, and (118) follows.

Remark. Notice that asymptotic expansions of higher orders of the density distribution $f(\lambda)$ which are available by the above method would readily generate the the corresponding asymptotic expansions for the moments $\mu_{r}$. 


\section{Concluding Remarks}

It was already mentioned that the contour of integration described in Section 6 plays a key role in the whole construction. In the case of functional (1) it helped to transform the integrals given explicitly in terms of Airy functions which emerged as solutions of certain differential equations. It turns out that the same contour can be used in many other similar cases, when the integrand is no more available explicitly, but is given as a certain Liouville-type asymptotics. In particular, in such a fashion one can obtain asymptotic expansions of the distributions of the functionals of the form

$$
\int_{0}^{1}|B(t)|^{\alpha} d t
$$

this possibility was indicated in Tolmatz (1992). It is understood that the same contour can provide asymptotics for a variety of Wiener functionals. Fatalov (2003) computed the asymptotics for (120) with $\alpha>0$ by a completely different method. It would be interesting to work out the method discussed in the present paper also for other Wiener functionals and to compare its scope with that of the alternative method due to Fatalov.

\section{References}

[1] Anderson, T. and Darling, D. (1952). Asymptotic theory of certain "goodness of fit" criteria based on stochastic processes. Ann. Math. Stat. 23 193-212.

[2] Borell, C. (1975). The Brunn-Minkowski inequality in Gauss space. Inventiones math. 30 207-216.

[3] Bruijn, N. G. de (1961). Asymptotic Methods in Analysis, 2nd ed. North-Holland Publishing Co.

[4] Cameron, R.H. and Martin, W.T. (1944). The Wiener measure of Hilbert neighborhoods in the space of real continuous functions. J. Math. Phys. XXIII 195-209.

[5] Cifarelli, D. M. (1975). Contributi intorno ad un test per l'omogenità tra due campioni. G. Econom. Ann. Econ. (N.S.) 34 233-249

[6] Fatalov, V.R. (2003). Asymptotics of large deviations of Gaussian processes of Wiener type for $L^{p}$ functionals, $p>0$, and the hypergeometric function. Sbornik: Mathematics 194:3 369-390.

[7] Johnson, B. McK. and Killeen, T. (1983). An explicit formula for the c.d.f. of the $L_{1}$ norm of the Brownian bridge. Ann. Probab. 11 807-808.

[8] Kac, M. (1946). On the average of a certain Wiener functional and a related limit theorem in calculus of probability. TAMS, $\mathbf{5 9} 401-414$.

[9] Kac, M. (1949a). On devations between theoretical and empirical distributions. Proc. Nat.Acad.Sci., 35 252-257.

[10] Kac, M. (1949b). On distribution of certain Wiener functionals. TAMS, 65 1-13.

[11] Kallianpur, G. and Oodaira, H. (1978). Freidlin-Wentzel type estimates for abstract Wiener spaces. Sankhya: The Indian Journal of Statistics, 40 Ser.A Pt.2 116-137. 
[12] Kiefer, J. K-sample analogues of the Kolmogorov-Smirnov and Cramér-von Mises tests. Ann. Math. Stat.. 30 420-447.

[13] Olver, F. W. J. (1974). Asymptotics and Special Functions. Academic Press, Inc.

[14] Prudnikov, A.P., et. al. (1990). Integrals and Series, vol. 3. Gordon and Breach Science Publishers.

[15] Rice, S. O. (1982). The integral of the absolute value of the pinned Wiener Process Calculation of its probability density by numerical integration. Ann. Probab. 10 240-243.

[16] Rosenblatt, M. (1951). On a class of Markov processes. TAMS, 71 120-135.

[17] Shepp, L. A. (1982). On the integral of the absolute value of the pinned Wiener process. Ann. Probab. 10 234-239. [Acknowledgement of priority 19 1397.]

[18] Takács, L. (1992). Random walk processes and their applications in order statistics. Ann. Appl. Probab. 2 435-459.

[19] Takács, L. (1993). On the distribution of the integral of the absolute value of the Brownian motion. Ann. Appl. Probab. 3 186-197.

[20] Tolmatz, L. (1992). Exact tail asymptotics of a certain Wiener functional. Ph.D. CWRU www.ohiolink.edu

[21] Tolmatz, L. (2000) Asymptotics of the Distribution of the Integral of the Absolute Value of the Brownian Bridge for Large Arguments. Ann. Probab. 28 132-139.

[22] Tolmatz, L. (2002) On the Distribution of the Square Integral of the Brownian Bridge. Ann. Probab. 30 253-269.

[23] Tolmatz, L. (2003) Addenda. On the Distribution of the Square Integral of the Brownian Bridge. Ann. Probab. 31 530-532. 